\title{
Bacterial Cellulose-Carboxymethyl Cellulose (BC:CMC) dry formulation as stabilizer and texturizing agent for surfactant-free cosmetic formulations
}

\author{
D. Martins, C. Rocha, F. Dourado, M. Gama * \\ CEB- Centre of Biological Engineering, Universidade do Minho, 4710-057 Braga, Portugal
}

G R A P H I C A L A B S T R A C T

Generic cosmetic creams stabilized with:

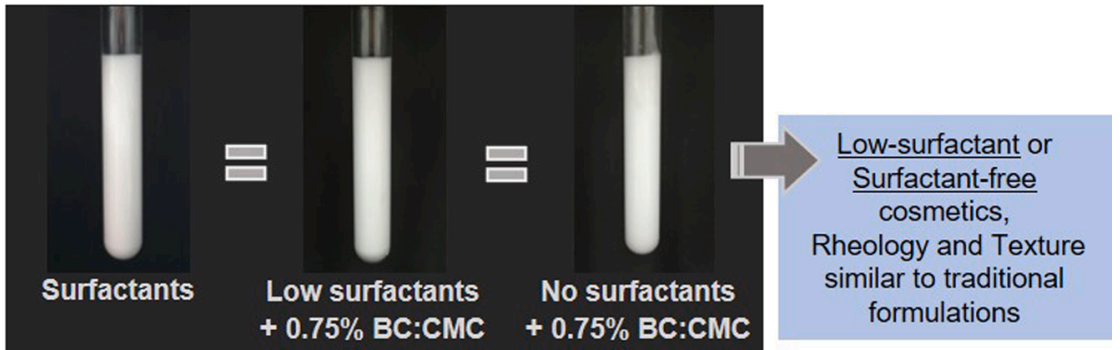

\section{A R T I C L E I N F O}

\section{Keywords:}

Pickering emulsions

Bacterial cellulose

Rheology

Texture analysis

\begin{abstract}
A B S T R A C T
Generic cosmetic creams (oil-in-water emulsions) were prepared using dry Bacterial Cellulose and Carboxymethyl Cellulose (BC:CMC) to study the possibility of partially or completely replacing surfactants, while ensuring a long-term stability and the required organoleptic characteristics. BC:CMC was benchmarked against two hydrocolloidal Avicel products (PC-591 and PC-611), commonly used as thickeners and stabilizing aids in cosmetics production. The emulsions were then characterized regarding storage stability, rheology, texture and microscopic features.

The full replacement of $5.5 \%$ surfactants with only $0.75 \%$ BC:CMC consistently showed similar results to those obtained with surfactants, namely concerning viscosity and texture. Although producing emulsions with larger oil droplets, BC:CMC provided for a very effective stabilization through a Pickering effect and by structuring the continuous phase. The more effective Avicel tested (PC-591) required a higher concentration (1.5\%) to achieve similar rheological profile but was ineffective in stabilizing the oil phase in a surfactant-free formulation with the adopted protocol. By replacing surfactants, dry BC:CMC matches a strong market need since both end users and manufacturers increasingly seek natural ingredients for cosmetic formulations.
\end{abstract}

\section{Introduction}

Cosmetic formulations are very complex mixtures, comprising water, oils, alcohols, active agents, preservatives, fragrances and others. Surfactants allow to balance all these components into a stable mixture. Besides their main role as emulsion stabilizers, surfactants also improve

\footnotetext{
* Corresponding author at: Departamento de Engenharia Biológica, Universidade do Minho, Campus de Gualtar, 4710-057 Braga, Portugal

E-mail address: fmgama@deb.uminho.pt (M. Gama).
} 
the rheological behaviour and texture of the formulations. In products such as cleansers, shampoos, washes and other personal hygiene products, they are the main ingredient, responsible for the cleaning function. Often surfactants can be irritating or sensitizing, depending on their chemical nature and concentration, originating or aggravating existent skin issues. Due to an increasing awareness and concern about these effects, more products are being formulated with milder or more natural surfactant systems, or partially replacing surfactants with polymeric thickeners such as xanthan gum, cellulose derivatives (e.g. hydroxypropyl methyl cellulose) and microcrystalline cellulose (MCC), among others. Thickeners help improving stability and rheology, and even bringing more pleasant sensory attributes to low-surfactant formulations [1-5].

MCC is already widely used in cosmetic products, often in combination with carboxymethyl cellulose (CMC). MCC particles can interact with each other in aqueous dispersions to form a network structure, after being subjected to high-energy mixing. MCC particles can help emulsification by Pickering stabilization; the three-dimensional polymer networks increase viscosity and create a solid-like rheological behaviour. Altogether, these properties help stabilizing emulsions and dispersions [6-9].

Cellulose from bacterial sources (e.g. Komagataeibacter genus) is chemically identical to wood or plant cellulose, but is obtained through fermentation in a pure state, consisting of long fibres with nanoscale thickness (high aspect ratio) [10,11]. Bacterial cellulose (BC) has already been studied notably in biomedical applications and also in the production of composites for the most diverse areas, the production and stabilization of emulsions and other food systems, optoelectronics and others [12-16].

In cosmetics, $\mathrm{BC}$ has been commercialized as face masks. The high water content and water retention capacity of the BC membrane can increase water uptake by the skin and improve hydration. Due to its high porosity, several drugs and active ingredients can be incorporated and released to skin under a controlled manner [17-21]. Other applications for $\mathrm{BC}$ as an ingredient for personal care and cosmetics have been studied and developed in recent decades, mostly found in patents. Examples include patent US 2007/019777 A1 [22] referring to mixtures of $\mathrm{BC}$ and $\mathrm{CMC}$ dried after alcohol precipitation (and spray-drying) for use as rheology modifier in a plethora of applications, including hair conditioners and hair styling products; patent WO 2011/019876 A2 [23] referring to personal cleansing formulations containing BC in combination with a cationic polymer as an external structuring system, to compensate for a smaller amount of internal structurants (surfactants); patent US 6534071 B1 [24] related to oil-in-water emulsions for cosmetic uses, free of surfactants, and containing cellulose fibrils, namely some commercial powdered BC formulations with $\mathrm{CMC}$; and others (WO 2011/056951 A1 [25], US 8716213 B2 [26]). These documents strongly demonstrate the growing commercial interest in $\mathrm{BC}$ for cosmetics and personal care market segments, but also a strong market need for BC products in their dry form. Indeed, dry products have advantages in transportation and storage since they occupy a smaller volume, plus are less prone to contaminations and can have longer shelf-life than hydrated forms.

Dry BC:CMC formulations have been recently reported by our group, which require low-energy mixing and short mixing time (under $5 \mathrm{~min}$ ) at room temperature, to achieve complete redispersion. These BC:CMC formulations display high viscosity and thickening power, ability to decrease oil/water interfacial tension and the capability to form threedimensional networks in aqueous media, acting as an external structurant. Even in low concentrations they are able to stabilize different heterogeneous systems for long periods of time, such as liquid-in-liquid emulsions (stability for over 90 days against coalescence and creaming, at $0.5 \%$ ) and solid-in-liquid dispersions (stability for over 4 days against sedimentation, at $0.15 \%$ ). Moreover, BC:CMC was able to outperform some commercially available MCCs in these systems [27,28].

Having proved the emulsifying, thickening and stabilizing capabilities of BC:CMC in a simple emulsion system, it is important to ascertain its performance in a complex matrix such as that of cosmetic products; it is also still necessary to validate its performance in the presence of - or in combination with - surfactants, once they serve a specific purpose in many products and are therefore indispensable. Furthermore, a surfactant substitute such as BC:CMC must not only stabilize cosmetic creams, it must also replicate relevant rheological and textural characteristics of traditional creams. This would allow to secure consumer acceptance, unlike what happens with other ingredients from natural sources.

In this work, generic cosmetic cream analogues (oil-in-water emulsions) have been prepared with dry BC:CMC in order to study the possibility of replacing, partially or completely, the surfactants in the cosmetic formulation, while maintaining a long-term stability and the characteristic rheological and organoleptic properties of the creams. A short-time mixing protocol was employed. Furthermore, a benchmark was made with MCC products that are optimized for cosmetic applications, to demonstrate that $\mathrm{BC}: \mathrm{CMC}$ can have a competitive advantage against some of the already marketed plant-based celluloses.

\section{Materials and methods}

\subsection{Materials and reagents}

BC wet membranes were supplied by HTK Food CO., Ltd (Ho Chi Minh, Vietnam).

Carboxymethyl cellulose ( $90 \mathrm{kDa}$, Degree of Substitution of 0.7) was supplied by Acros Organics (Geel, Belgium). Isohexadecane (Purolan IHD) was gently provided by Lanxess (Leverkusen, Germany). Steareth 21 (Brij S721) was purchased from Croda (Goole, United Kingdom). Liquid paraffin was provided by Labchem (Loures, Portugal). Butylene glycol, Dimethicone, Stearic acid, Steareth 2 (Brij S2), Nile Red, Calcofluor White and Sodium azide were purchased from Sigma (St. Louis, Missouri, USA).

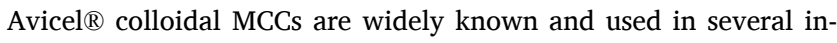
dustry fields. For this work, two were chosen for being specifically indicated for cosmetic applications, and used as received: Avicel PC-611 (low viscosity, containing 11.3-18.8\% CMC) and Avicel PC-591 (medium viscosity, containing 9.0-15.0\% CMC), kindly provided by DuPont (Wilmington, Delaware, USA).

\subsection{BC:CMC preparation}

Dried BC:CMC was prepared using a methodology adapted from Martins et al. [27]. BC membranes were left in $0.1 \mathrm{M} \mathrm{NaOH}$ solution for 4 days (with daily solution exchange), at room temperature, then washed thoroughly with distilled water until the $\mathrm{pH}$ was that of the distilled water. The washed membranes were then wet ground using first a fixed speed hand blender (Sammic blender TR250, Sammic S. L.) at $9000 \mathrm{rpm}$ until a homogeneous pulp was obtained, and then using a high-speed blender (Moulinex Ultrablend $1500 \mathrm{~W}$ ) at $24000 \mathrm{rpm}$ for 1 min. The solid fraction of the obtained pulp was determined by dry weight and adjusted to $0.5 \%(\mathrm{~m} / \mathrm{v})$. To this pulp, the same volume of 0.5 $\%(\mathrm{~m} / \mathrm{v}) \mathrm{CMC}(90 \mathrm{kDa})$ was added, making up a 1:1 mixture (BC:CMC) of $0.5 \%$ total solids. This mixture was left under magnetic stirring overnight and autoclaved at $121{ }^{\circ} \mathrm{C}$ for $20 \mathrm{~min}$. The formulation was freeze-dried for 5 days at $-100{ }^{\circ} \mathrm{C}$ and approximately 0.05 mbar and stored in a desiccator until use. A similar BC:CMC material (spray-dried) has been previously characterized [27], showing Zeta potential of (-67.0 $\pm 3.9) \mathrm{mV}$ and a mean diameter, $\operatorname{Dv}(50)$, of $(601 \pm 19.7) \mu \mathrm{m}$ in aqueous suspension.

\subsection{Emulsions preparation}

Generic cosmetic cream emulsions were prepared (Table 1) in triplicate, using a methodology adapted from Gilbert et al. [29-31], where 
Table 1

Composition, in mass fraction $(\% \mathrm{w} / \mathrm{w})$, of the generic cosmetic cream emulsions.

\begin{tabular}{|c|c|c|c|c|c|c|c|c|c|c|c|c|}
\hline \multirow[b]{3}{*}{ Polymer } & \multirow{3}{*}{$\frac{\mathrm{FF}^{*}}{-}$} & \multicolumn{7}{|c|}{ Low surfactants formulations (LSF) } & \multicolumn{4}{|c|}{ No surfactants formulations (NSF) } \\
\hline & & \multirow{2}{*}{$\begin{array}{l}\text { LS Control } \\
-\end{array}$} & \multicolumn{2}{|c|}{ LS BC:CMC } & \multicolumn{2}{|c|}{ LS PC-591 } & \multicolumn{2}{|c|}{ LS PC-611 } & \multicolumn{2}{|c|}{ NS BC:CMC } & \multicolumn{2}{|c|}{ NS PC-591 } \\
\hline & & & 0.5 & 0.75 & 0.75 & 1.5 & 0.75 & 1.5 & 0.5 & 0.75 & 0.75 & 1.5 \\
\hline Distilled water & 81 & 85.95 & 85.45 & 85.2 & 85.2 & 84.45 & 85.2 & 84.45 & 86 & 85.75 & 85.75 & 85 \\
\hline Butylene glycol & 4 & 4 & 4 & 4 & 4 & 4 & 4 & 4 & 4 & 4 & 4 & 4 \\
\hline Steareth2 & 3 & 0.3 & 0.3 & 0.3 & 0.3 & 0.3 & 0.3 & 0.3 & - & - & - & - \\
\hline Steareth 21 & 2 & 0.2 & 0.2 & 0.2 & 0.2 & 0.2 & 0.2 & 0.2 & - & - & - & - \\
\hline Stearic acid & 0.5 & 0.05 & 0.05 & 0.05 & 0.05 & 0.05 & 0.05 & 0.05 & - & - & - & - \\
\hline Isohexadecane & 7 & 7 & 7 & 7 & 7 & 7 & 7 & 7 & 7 & 7 & 7 & 7 \\
\hline Dimethicone & 1 & 1 & 1 & 1 & 1 & 1 & 1 & 1 & 1 & 1 & 1 & 1 \\
\hline Paraffin & 1.5 & 1.5 & 1.5 & 1.5 & 1.5 & 1.5 & 1.5 & 1.5 & 1.5 & 1.5 & 1.5 & 1.5 \\
\hline
\end{tabular}

* Full formulation of the generic cream, prepared according to Gilbert et al. [29-31].

Steareth 2, Steareth 21 (non-ionic) and stearic acid were used as surfactants. The full formulation (FF), as described in the cited literature is displayed in Table 1, containing a total surfactants concentration of 5.5 $\%$ (in mass). Samples with added polymers (BC:CMC or MCC) were prepared with only $10 \%$ of that amount $(0.55 \%$ of total surfactants) and designated "Low Surfactant Formulations" (LSF). This concentration was chosen after several exploratory tests, for lying below the stability threshold of the emulsion (which was determined to be around $1.38 \%$ of total surfactants, where creaming started to occur). A low surfactant control ("LS Control") cream was also prepared without any polymer (the amount of polymer was substituted by distilled water).

Furthermore, to investigate the stabilizing properties of the polymers alone, emulsions were also prepared without surfactants (designated "No Surfactant Formulations", NSF). No control was made in this case since emulsions could not be obtained.

It has been previously reported that a dry formulation of $\mathrm{BC}: \mathrm{CMC}$ at a concentration of $0.5 \%$ was capable of fully stabilizing a $10 \%$ isohexadecane emulsion up to 90 days [27]. Therefore, for this work with a similar material, a concentration of $0.5 \%$ BC:CMC was chosen; additionally, a concentration of $0.75 \% \mathrm{BC}: \mathrm{CMC}$ was also tested in order to increase the viscosity and possibly improve the textural profile of the cosmetic cream formulation. On the other hand, MCCs usually require higher amounts to create a structuring network within the sample, up to $1.5 \%$ [9]; besides, a lower concentration of $0.75 \%$ was also tested for Avicels to have a comparison point with BC:CMC.

BC:CMC, Avicel PC-591 and Avicel PC-611 were pre-dispersed in half of the volume of water used in each formulation, with magnetic stirring for $10 \mathrm{~min}$ at $700 \mathrm{rpm}$, at room temperature.

For the FF and LSF samples, the oil phase components and the surfactants were firstly mixed together in a magnetic stirrer at $200 \mathrm{rpm}$ and approximately $75^{\circ} \mathrm{C}$, to melt the surfactants into the oils. Warm distilled water and butylene glycol were then added to the oil phase under vigorous stirring $(700 \mathrm{rpm})$ for $2 \mathrm{~min}$, promoting a primary coarse emulsion, also at approximately $75^{\circ} \mathrm{C}$. Afterwards, for the LSF samples, the pre-dispersed polymer was added under continuous agitation, and mixed for $1 \mathrm{~min}$ more. Each sample (FF and LSF) was then immediately emulsified for $2 \mathrm{~min}$ at $15000 \mathrm{rpm}$ in a T 25 digital Ultra-turrax (IKA, Germany) with a S25 N-18 G dispersing tool. Finally, the emulsions were left to cool to room temperature, under magnetic stirring.

For the NSF samples, oil phase components were mixed under magnetic stirring at $200 \mathrm{rpm}$ and room temperature. The polymers were pre-dispersed in the total amount of distilled water and butylene glycol was added. This aqueous phase was stirred vigorously with the oil phase and emulsified in the rotor-stator homogenizer as previously described.

Emulsions of $100 \mathrm{~g}$ were prepared in triplicate for each condition. Sodium azide $(0.02 \%)$ was then added to all emulsions to prevent microbial contamination, and the samples were stored at room temperature in well-sealed containers to prevent water evaporation until analysis.

\subsection{Evaluation of stability}

The samples' stability over time, at room temperature, was assessed by visual inspection and optical microscopy. An aliquot of $10 \mathrm{~mL}$ of each sample was transferred into a tube just after emulsion preparation and kept sealed. Photographs were taken after 1 and 30 days of preparation. For microscopic analyses, a drop of each emulsion was placed on a glass slide, coversliped and visualized on bright field in an Olympus BX51 fluorescence microscope with Cellsense software and 10X magnification objective lens (Olympus Europa SE \& Co. KG, Hamburg, Germany).

\subsection{Rheological analysis of the emulsions}

Rheological tests were performed in a DHR-1 controlled stress rheometer with Trios version 4 software for Windows (TA Instruments, New Castle, Delaware, USA) at $25{ }^{\circ} \mathrm{C}$ using a cone and plate geometry ( $60 \mathrm{~mm}, 2.006^{\circ}$ cone angle, $64 \mu \mathrm{m}$ gap). In a flow sweep test, samples shear stress and viscosity were measured at varying shear rate. Shear rate was increased from $0.01 \mathrm{~s}^{-1}$ to $1000 \mathrm{~s}^{-1}$, then decreased to $0.01 \mathrm{~s}^{-1}$, and finally increased once again to $1000 \mathrm{~s}^{-1}$ (logarithmic mode, 5 points per decade), and the values from the first and third sweeps were considered for analysis. Results were plotted as the average and standard deviation of the triplicate samples.

A Three Interval Thixotropy Test (3ITT) was performed in controlled shear rate mode to investigate the time-dependent behaviour of the formulations, through monitoring the samples' viscosity. Samples were deformed with a shear rate of $0.1 \mathrm{~s}^{-1}$ for $150 \mathrm{~s}$ in the first interval, 300 $\mathrm{s}^{-1}$ for $60 \mathrm{~s}$ in the second interval, and finally back to $0.1 \mathrm{~s}^{-1}$ for $600 \mathrm{~s}$ to allow for structure recovery.

An oscillatory strain sweep test was carried out to determine the Linear Viscoelastic Region (LVR) of the different samples. Frequency was set at $1 \mathrm{rad} / \mathrm{s}$ and strain amplitude was increased from $0.02 \%$ to 500 $\%$. An oscillatory frequency sweep test was performed (after a pre-shear from $1 \mathrm{~s}^{-1}$ to $100 \mathrm{~s}^{-1}$, and $100 \mathrm{~s}^{-1}$ to $1 \mathrm{~s}^{-1}$ ), with angular frequency ranging from 0.05 to $200 \mathrm{rad} / \mathrm{s}$ at a strain of $0.3 \%$, within the LVR as determined from the previous strain sweep test. Results were plotted as the average and standard deviation of the triplicate samples.

Cream emulsions were lastly monitored for changes in the rheological behaviour while subjected to oscillatory temperature cycles. Silicon oil was used to prevent solvent evaporation. The storage modulus was recorded as samples were cooled and heated between $10^{\circ} \mathrm{C}$ and $50{ }^{\circ} \mathrm{C}$, at a constant angular frequency of $10 \mathrm{rad} / \mathrm{s}$ and $0.3 \%$ strain, according to the following steps: (1) initial hold at $10^{\circ} \mathrm{C}, 240 \mathrm{~s}$; (2) temperature ramp from $10^{\circ} \mathrm{C}$ to $50^{\circ} \mathrm{C}$ at a heating rate of $5{ }^{\circ} \mathrm{C} / \mathrm{min}$, followed by a soak time of $600 \mathrm{~s}$ at $50{ }^{\circ} \mathrm{C}$; (3) temperature ramp from $50{ }^{\circ} \mathrm{C}$ to $10^{\circ} \mathrm{C}$ at a cooling rate of $5{ }^{\circ} \mathrm{C} / \mathrm{min}$, followed by a soak time of $600 \mathrm{~s}$ at $10{ }^{\circ} \mathrm{C}$; (4) repeat step 2 ; (5) repeat step 3.

\subsection{Textural analysis}

Texture analysis was performed in a TA.HD Plus Texture analyser 
(Stable Micro Systems, Godalming, United Kingdom) with a $5 \mathrm{~kg}$ load cell. Samples were analysed in cylindrical cups $(50 \mathrm{~mm}$ diameter, $75 \mathrm{~mm}$ height), and sample height was $35 \mathrm{~mm}$.

A Penetration test was made with a $\mathrm{P} / 10$ probe, pre-test speed of 0.5 $\mathrm{mm} / \mathrm{s}$, test speed of $2 \mathrm{~mm} / \mathrm{s}$ and post-test speed of $2 \mathrm{~mm} / \mathrm{s}$. The trigger force was set at $0.5 \mathrm{~g}$, and the probe penetrated $20 \mathrm{~mm}$ into the sample. Firmness (the maximum penetration force) and consistency (the work of penetration, area below the curve up to the point of the maximum force) were automatically calculated by the Exponent software. Results were calculated as the mean and standard deviation of the triplicate samples for each condition.

For the Back-extrusion test, a $45 \mathrm{~mm}$ diameter disc was used; pre-test speed was $1 \mathrm{~mm} / \mathrm{s}$, test speed was $1 \mathrm{~mm} / \mathrm{s}$ and post-test speed was 2 $\mathrm{mm} / \mathrm{s}$. The trigger force was set at $0.5 \mathrm{~g}$ and the disc penetrated $35 \mathrm{~mm}$ into the sample. Firmness, consistency, cohesiveness (maximum negative force) and index of viscosity (or 'work of cohesion', the area of the negative region of the curve that represents resistance to withdrawal) were automatically calculated by the Exponent software version 6 for Windows (Stable Micro Systems). Results were calculated as the mean and standard deviation of the triplicate samples for each condition.

Statistical analysis was performed using GraphPad Prism version 5 for Windows (GraphPad Software Inc., San Diego, California, USA). Results were expressed as mean \pm standard deviation. A one-way analysis of variance (ANOVA) was performed, followed by Dunnett's Multiple Comparison Test, to establish the significance of differences.

\subsection{Confocal Laser Scanning Microscopy (CLSM)}

Before observation, aliquots of emulsions were stained with $1 \%$ of Nile Red solution $(0.5 \mathrm{mg} / \mathrm{mL}$ in acetone) and $4 \%$ of Calcofluor White stain solution $(0.02 \mathrm{mg} / \mathrm{mL}$ in distilled water). A sample of $10 \mu \mathrm{L}$ was placed on a glass slide and coverslipped. Observation was made in a Confocal Laser Scanning Microscope Olympus BX61, model FluoView FV1000 (version 4), with an objective lens of 10X magnification (Olympus Europa SE \& Co. KG).

The excitation and emission wavelengths used for Nile red detection were $559 \mathrm{~nm}$ and $592 \mathrm{~nm}$, respectively, and for Calcofluor white stain were $405 \mathrm{~nm}$ and $461 \mathrm{~nm}$, respectively.

\section{Results and discussion}

\subsection{Evaluation of stability over time}

The visual aspect of the cosmetic emulsions was recorded photographically at 1 and 30 days after preparation (Fig. 1), at room temperature. Phase separation and creaming effects were observed in some cases, pointed out in Fig. 1 with red arrows. In the case of the LSF, the addition of Avicel PC-611 did not effectively stabilize the emulsions. These samples started to show instability 1 day after preparation. The more concentrated samples (LS 1.5 PC-611) showed a small but visible creaming, remaining unaltered until the 30th day of storage, while the ones with lower concentration (LS 0.75 PC-611) exhibited very pronounced creaming and even sedimentation. Creaming is the rise of the disperse phase droplets to the top of the emulsion, a phenomenon that depends on droplet size, viscosity of the continuous phase and density differences between continuous and dispersed phases [32]. In this case, the observation of creaming suggests low viscosity and/or insufficient continuous phase structuring in these samples, a polymer network that would immobilize the oil droplets and prevent their buoyancy driven by gravity. This will be further evaluated in the section regarding rheology experiments.

No oil separation was visible because the small amount of surfactants in the emulsions was sufficient to stabilize the oil phase, as can be understood from the LS Control assay. For BC:CMC and Avicel PC-591, at both concentrations, emulsions remained fully stable as no creaming nor phase separation were observed. It has been previously shown that dry BC:CMC has the ability to reduce the oil/water interfacial tension [27, 28], a property associated to emulsifying agents. Therefore, emulsions were also prepared without surfactants (NSF), to evaluate the effect of the cellulose products alone in the stabilization of the oil phase. With NS Avicel PC-611, even at $1.5 \%$, phase separation occurred immediately after preparation, so this assay was discontinued. Avicel PC-591 was also incapable of stabilizing the emulsions, in the conditions of the adopted protocol (at a concentration of $1.5 \%$ there was a visible line of oil at the surface of the emulsion). Contrarily, the emulsions with BC:CMC, at both tested concentrations, showed stability throughout the 30-day storage period, without visually detectable changes. Due to the pronounced instability of the emulsions prepared with Avicel PC-611, only the LS 1.5

\begin{tabular}{|c|c|c|c|c|c|c|c|c|c|c|c|}
\hline \multirow[b]{3}{*}{ FF } & \multicolumn{7}{|c|}{ Low surfactant formulations (LSF) } & \multicolumn{4}{|c|}{ No surfactant formulations (NSF) } \\
\hline & & \multicolumn{2}{|c|}{$\mathrm{BC}: \mathrm{CMC}$} & \multicolumn{2}{|c|}{ Avicel PC-591 } & \multicolumn{2}{|c|}{ Avicel PC-611 } & \multicolumn{2}{|c|}{$\mathrm{BC}: \mathrm{CMC}$} & \multicolumn{2}{|c|}{ Avicel PC-591 } \\
\hline & Control & $0.5 \%$ & $0.75 \%$ & $0.75 \%$ & $1.5 \%$ & $0.75 \%$ & $1.5 \%$ & $0.5 \%$ & $0.75 \%$ & $0.75 \%$ & $1.5 \%$ \\
\hline
\end{tabular}
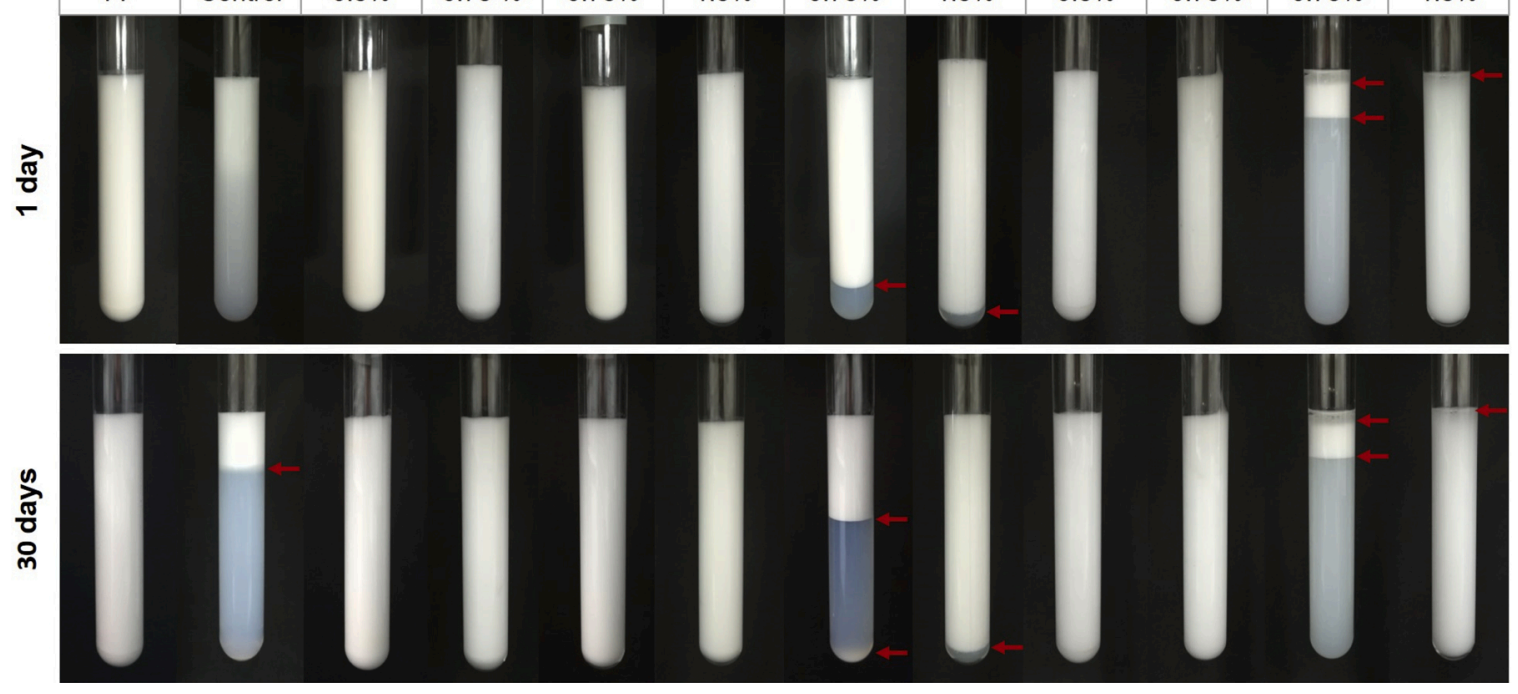

Fig. 1. Photographs of the cosmetic emulsions stabilized with different concentrations of BC:CMC, Avicel PC-591 and Avicel PC-611, taken 1 day after preparation and after 30 days of storage, at room temperature. Red arrows indicate visible lines of phase separation, creaming effect or sedimentation. 
PC-611 was included in the following analyses; the condition NS 0.75 PC-591 was also disregarded.

In many cosmetic emulsions, surfactants are the main emulsifier/ stabilizer and additional thickeners only serve rheological and textural purposes. In Fig. 2 it is visible that the emulsion droplet sizes increase with the decrease in added surfactants and their replacement with polymers: $\mathrm{FF}$ emulsion has an almost homogeneous microscopic appearance; LSF show some larger droplets than FF; and then NSF show even larger droplets, more heterogeneous in size (broad distribution). In $\mathrm{FF}$, higher surfactant concentration allows for a larger oil-water interfacial area and reduced interfacial tension, resulting in decreased oil droplet size.

In LSF, surfactants are still the main emulsifying agent, as the oil is much better dispersed than in the corresponding NSF (where oil globules reach several tens of micrometers). However, as a proof of concept, this experiment allowed us to further demonstrate that BC:CMC alone is capable of stabilizing the oil phase of a generic cosmetic emulsion.

Fig. 2 also shows the microscopic evolution of the emulsions over time, comparing images obtained 1 and 30 days after preparation. No significant changes in the microstructure are apparent during the storage period as there is no significant variation in the emulsion size distribution, confirming the emulsions' stability over time. This is most relevant in the case of the NS BC:CMC formulations, which display larger, yet well dispersed droplets even after 30 days, no noticeable evolution in size being detected. The significant size of the oil droplets did not translate in a loss of stability - a particular characteristic of Pickering emulsions. Particle-stabilized emulsions often have larger
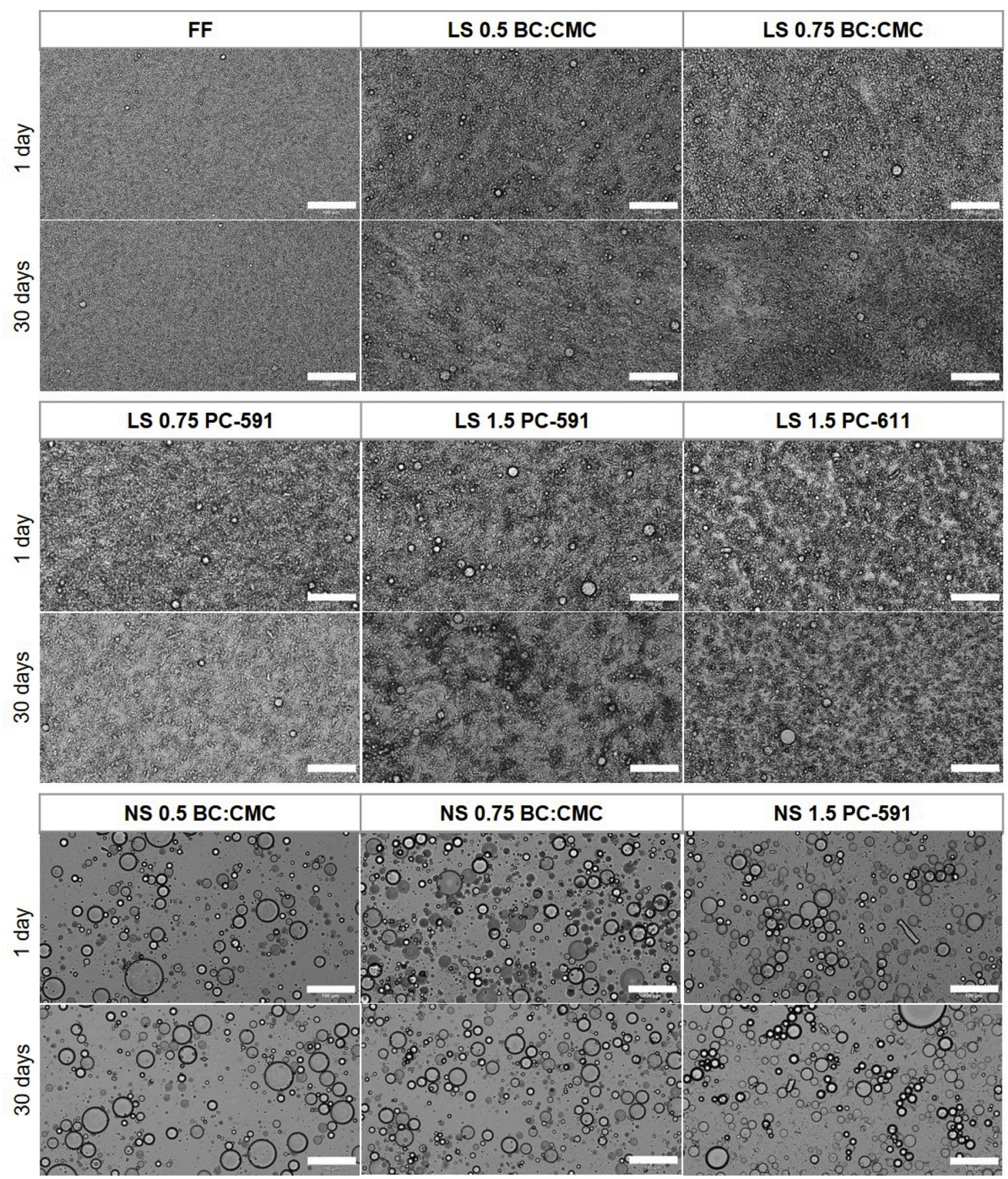

Fig. 2. Optical micrographs (10X magnification) of the cosmetic emulsions stabilized with different concentrations of BC:CMC, Avicel PC-591 and Avicel PC-611, taken after a storage time of 1 day and 30 days, at room temperature. Scale bars correspond to $100 \mu \mathrm{m}$. 
droplet sizes (at times reaching few millimeters) that, contrarily to surfactant-stabilized emulsions, are very stable against coalescence due to the steric (mechanical) barrier created by the solid particles adsorbed around the oil droplets [33-35].

By CLSM (Fig. 3), the spatial distribution of the fibres in a NSF, namely NS 0.5 BC:CMC, was investigated. The BC fibres (stained in blue) can be seen all around the oil droplets (stained in red). This is more clearly visible in the bottom section of the scan, where arrows point out a more intense blue fibre network covering oil droplets. As the scan moves upwards on the sample, in the middle section a blue halo is also evident around the oil droplets, in the oil/water interface (pointed by arrows). These observations support the Pickering emulsion mechanism in the NS BC:CMC. Additionally, BC fibres can be seen in all images in the bulk of the aqueous phase, creating a dense 3D network probably responsible for stabilizing the droplets in suspension and preventing their coalescence.

\subsection{Rheological assessment}

Fig. 4 shows that the different tested celluloses in aqueous suspensions present quite distinct viscosity profiles (from higher to lower): BC: CMC, Avicel PC-591 and Avicel PC-611 (the two Avicel samples have medium and low viscosity, respectively, as reported by the supplier). All dispersions showed a shear thinning behaviour, widely common in complex fluids, resulting from the progressive entanglement breakdown. This effect is sharper for BC:CMC mixtures and much smoother for PC-611, and can be associated with different degrees of hydrogen bonding. Since BC fibres are much longer and thinner (in the order of nanometres) than microcrystalline cellulose, they have a higher aspect ratio and specific surface area [11]. For this reason they are more flexible, which favours interaction points between them via hydrogen bonds. MCC particles are crystals of larger diameter, having smaller specific surface area and lower flexibility, so there are fewer possibilities for interaction. A concentration effect is also observed in the results of
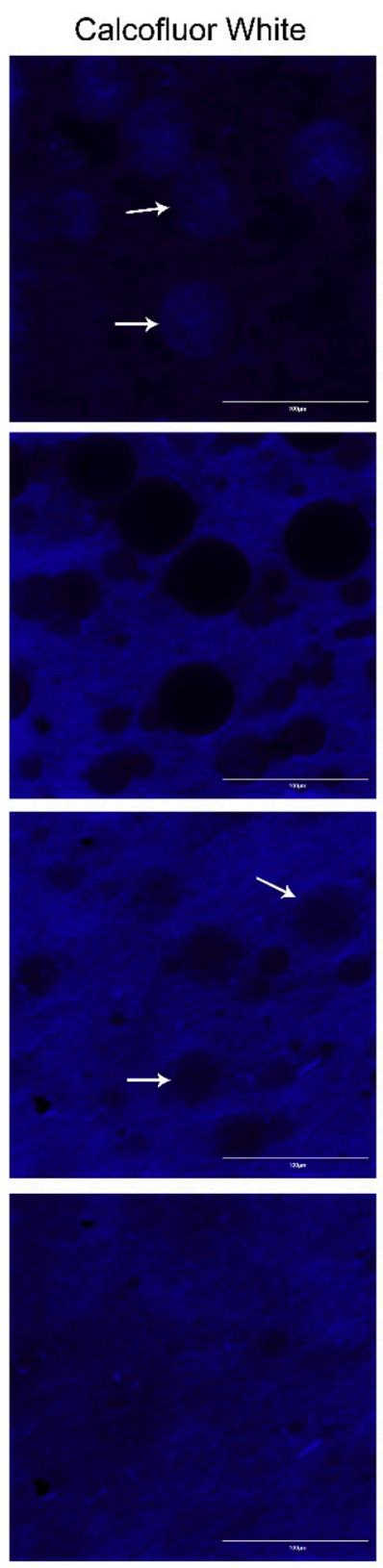

Nile Red
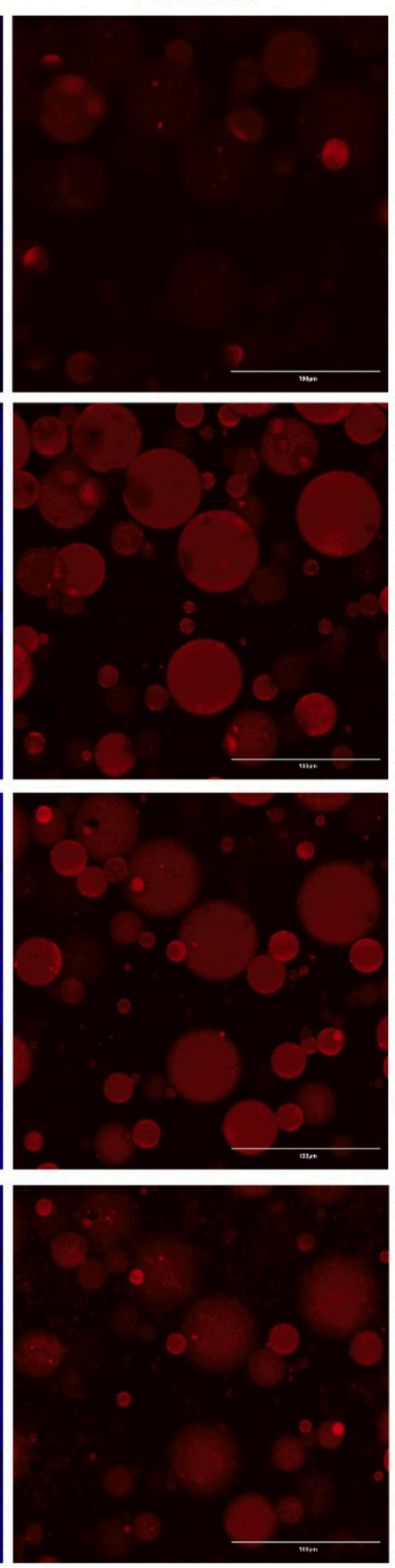

Merge
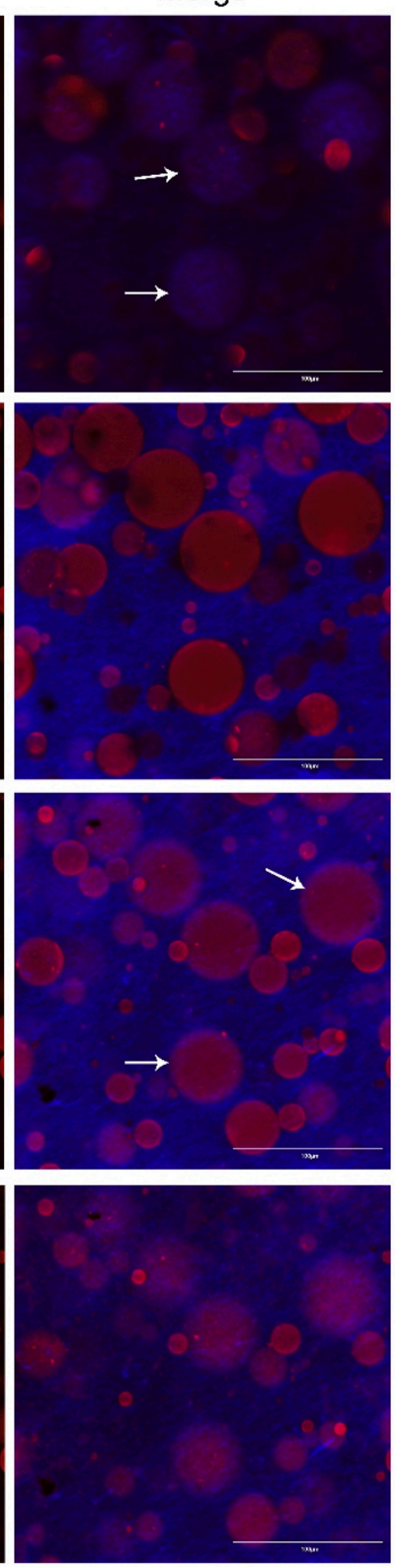

\section{Bottom \\ section}
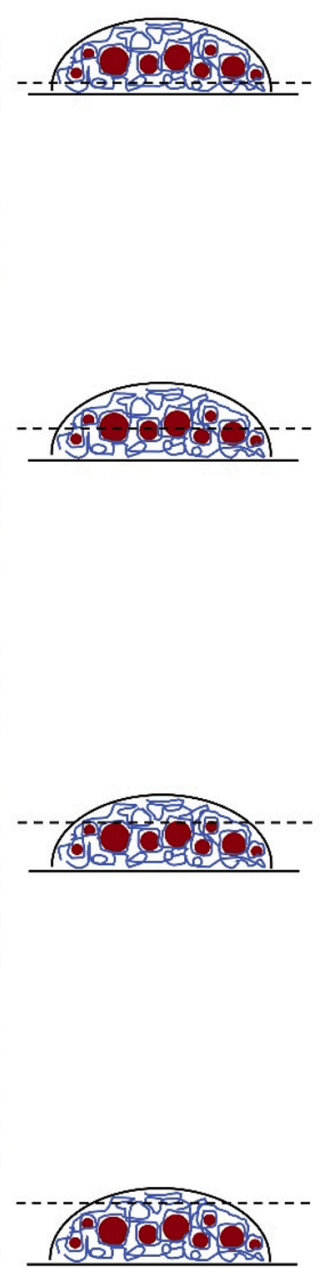

Top

section

Fig. 3. CLSM micrographs (10x magnification) of NS 0.5 BC:CMC. Oil phase is stained in red by Nile Red dye, and BC fibers are stained blue by Calcofluor White stain. Scale bars correspond to $100 \mu \mathrm{m}$. 


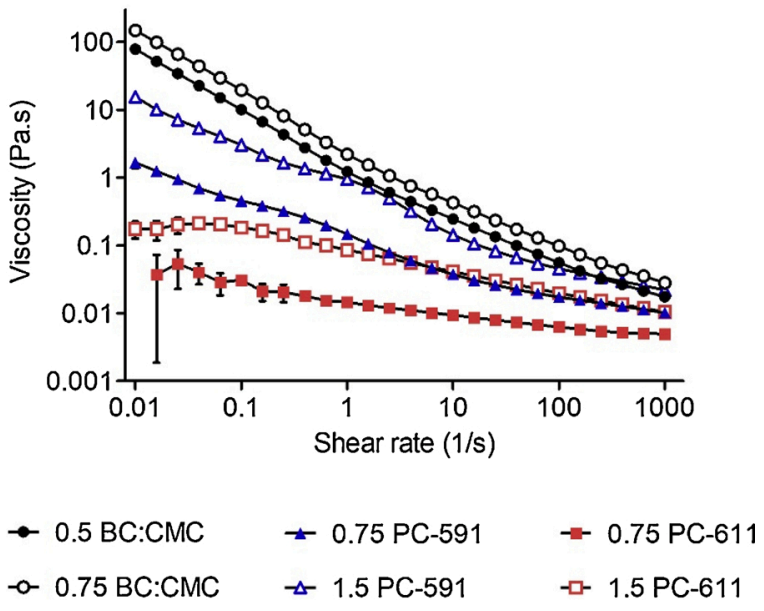

Fig. 4. Flow curves of aqueous dispersions of the polymers used in this study, at different concentrations. Results are the average of triplicate samples, obtained from the third consecutive flow sweep (FS3). Bars represent standard deviation.

Fig. 4, usually ascribed to the presence of more entanglement points [36].

\subsubsection{Low surfactant formulations}

Results of the first (FS1) and third (FS3) consecutive flow-sweeps of the emulsions are shown in Fig. 5a and b, respectively. The first flow

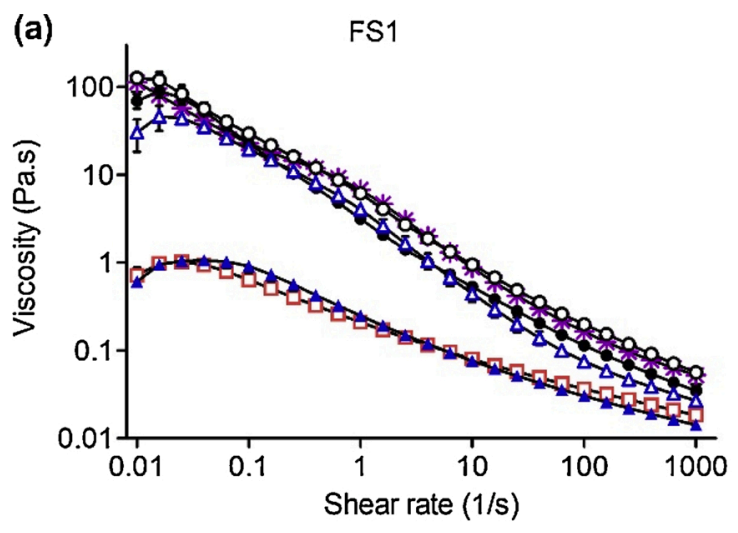

(c)

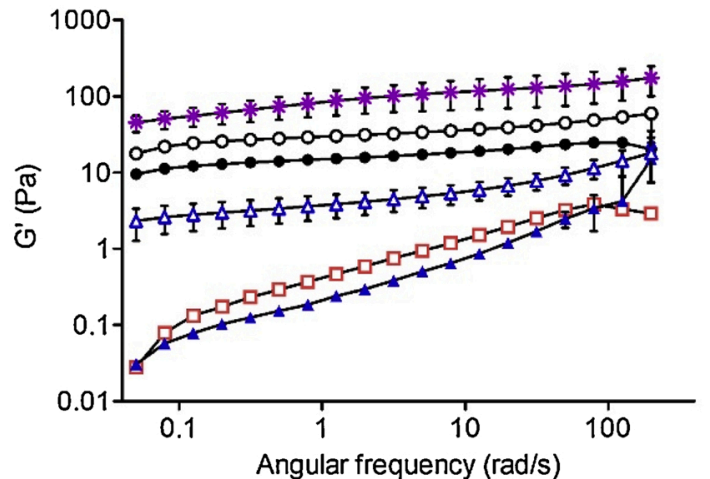

sweep emulates flow behaviour of the samples after storage in commercial recipients. The purpose of the third flow-sweep is to investigate the stability of the formulations after undergoing multiple shearing steps, removing forces that might have built up during storage and revealing more of the intrinsic structure of the emulsion.

From Fig. 5 (a and b), all LSF and FF samples viscosities decreased with the increase in the shear rate, characteristic of a shear-thinning (pseudoplastic) behaviour. This is an important property for cosmetic creams, as when applying the cream on the skin (under shearing force applied by hand) the viscosity decreases and spreading is facilitated. Shear rates involved in rubbing creams or lotions on the skin can vary from $10^{2}$ to $10^{4} \mathrm{~s}^{-1}$; on the other hand, draining under gravity corresponds to shear rates between $10^{-1}$ and $10^{1} \mathrm{~s}^{-1}$, and sedimentation of particles in a suspending liquid happens below $10^{-3} \mathrm{~s}^{-1}$ [37]. In the LSF samples the viscosity also increases with the respective polymer's concentration. The emulsions prepared with BC:CMC present similar shear viscosity values to those of its aqueous suspensions (Fig. 4). Thus, for these samples it can be said that the surfactants (present in low concentration - $10 \%$ relatively to the control) were not playing the major viscosifying role and that the overall viscosity pattern is controlled by the continuous phase: an aqueous network of cellulosic particles which may interact cohesively by hydrogen bonds while repulsive electrostatic forces arising from the presence of the polyelectrolyte (CMC) counterbalances these forces, promoting the full spreading or swelling of the fibres, strongly contributing to the structuring effect. In this case the different oil droplet patterns do not seem to lead to differences in the interaction between droplets that could interfere with the viscosity (e.g. through the formation of droplet clusters), or these differences are

\section{(b) FS3}

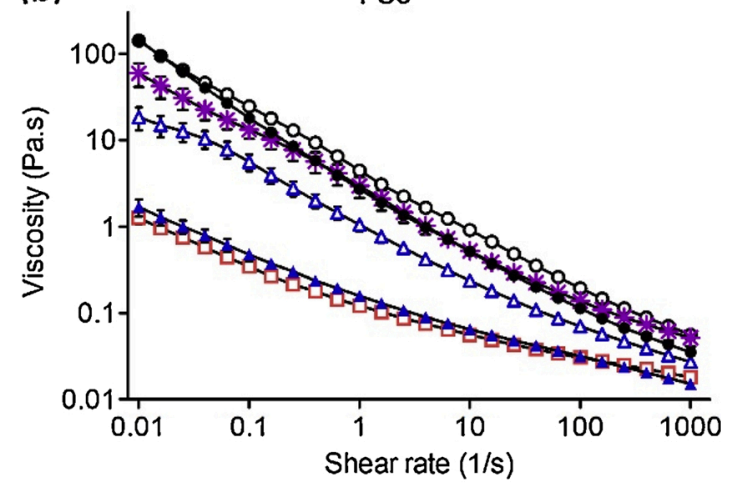

(d)

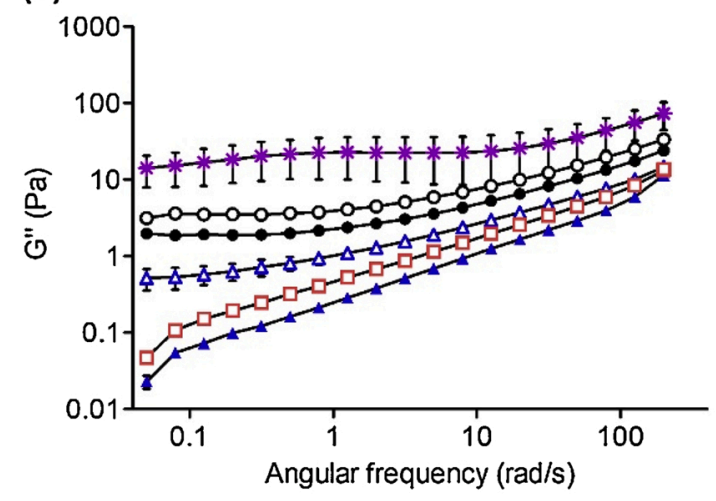

$$
\begin{array}{lll}
\text { * FF } & \rightarrow \text { LS 0.5 BC:CMC } & \rightarrow-\text { LS 0.75 BC:CMC } \\
\text { - LS 0.75 PC-591 } & \rightarrow \text { LS 1.5 PC-591 } & - \text { - LS 1.5 PC-611 }
\end{array}
$$

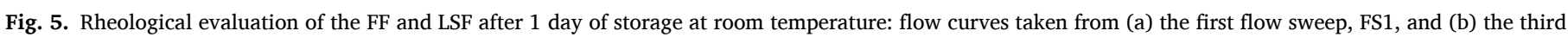

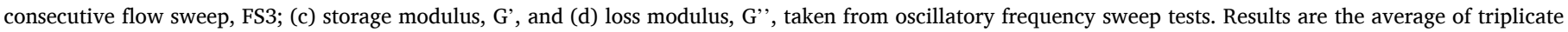
samples. Bars represent standard deviation. 
overlapped by the continuous phase governance.

On the other hand, FF does not contain any polymer, so its viscosity is related to the microstructure of the emulsion: influence of the surfactant, size distribution of the oil droplets and their interaction, which offer resistance to movement under shearing.

The flow curves of LSF PC-591 and PC-611 show higher viscosity in comparison to the respective polymers' suspensions. The overall flow profile in this case depends on both the viscosity of the suspended solid particles (which is relatively low), but also on the microstructure of the emulsion - a combination of continuous and dispersed phase contributions.

As seen in Fig. 5a, the FF shows a tendency of ever-increasing viscosity towards lower shear rates, while the cellulose-stabilized LSF creams demonstrate a plateau or slight increase of viscosity in the first points of the graphic, until a critical shear rate (below $0.1 \mathrm{~s}^{-1}$ ). This may indicate that in the range of very low shear rates, the disordered entangled network is only slightly disturbed and is able to recover. Despite this narrow initial plateau, most samples' behaviour approximate the FF for the rest of the shear rate interval tested, with a disordered entangled network disruption rate higher than the recovery rate resulting in the decrease of the viscosity with the increase of shear rate. Both concentrations of BC:CMC were able to thicken the samples to a viscosity close to FF (LS 0.5 BC:CMC) or identical (LS 0.75 BC:CMC). The LS 1.5 PC-591 sample also has a viscosity profile that approximates to FF around $0.1 \mathrm{~s}^{-1}$, with a lower viscosity profile onwards. Further, the third flow sweep (Fig. 5b) reveals that this sample loses viscosity after shearing, so the higher apparent initial viscosity could be due to weak interactions between the components, built up during rest time, that were eliminated by the application of a shear force. A small decrease in viscosity was also observed in the FF; samples with BC:CMC showed high structural integrity even after the multiple shearing cycles, maintaining a high viscosity. The viscosity plateau disappeared in the third flow sweep for all the polymer-stabilized emulsions.

At low shear rates, below $0.1 \mathrm{~s}^{-1}$, the average viscosity of the samples with $0.5 \% \mathrm{BC}$ :CMC is not much different from the $0.75 \%$ ones. This behaviour changed after 30 days of storage (see Appendix A.1), the differences at low shear rates becoming more defined between the two concentrations, as in the polymers' viscosity graphic of Fig. 4 .

Creams prepared with Avicel PC-611 and PC-591 could not match the rheological profile of the control even at $1.5 \%$, always showing lower viscosities. These MCCs, at the concentrations tested, could not provide the necessary viscosity in a formulation with reduced surfactants. Comparing both Avicel at the same concentration (0.75\%) with $\mathrm{BC}$ :CMC, the later had much higher thickening power, as could be already induced from the behaviour of the dispersions of the polymer mixtures alone.

Regarding the dynamic tests, the storage/elastic modulus (G') and loss/viscous modulus (G') of the samples were analysed. Solid materials are characterized by an elastic behaviour, whereas fluid materials have viscous behaviour. Viscoelastic/pseudoplastic materials such as polymeric dispersions or emulsions are neither true fluids nor solids by definition, but share behavioural characteristics of both - an elastic component represented by G', and a viscous component represented by G' [36,37]. Materials with a more pronounced solid behaviour component are more capable of keeping particles in suspension, which in this case are oil droplets. The materials with the largest solid component are, in theory, more stable.

Results from the oscillatory strain sweep tests are reported in Appendix A.3. The emulsion LS 1.5 PC-611 shows a predominant viscous behavior throughout the entire strain interval, which is in accordance with the creaming effect verified in Fig. 1 due to insufficient structuring of the continuous phase. LS 0.75 PC-591 also displays a weak phase structuring, with nearly G' $=$ G' ' until $10 \%$ strain, from where G' further decreases. All the other samples show a main elastic behavior at the LVR, but varying on the critical strain and the G'/G' crossing point. The critical point is when the viscoelastic material's rheological properties are no longer independent on the strain: where the LVR ends, and structure disruption starts. With further increasing strain, G' will decrease. At some point, it will become lower than G' and the behavior of the material changes to fluid-like. In the FF cream this happens below $10 \%$ strain, while for the LS and NS BC:CMC and the LS 1.5 PC-591 emulsions the critical strain is higher than $10 \%$ and the crossover point only occurs around $100 \%$ strain.

Regarding now the oscillatory frequency sweep tests, conducted at a strain of $0.3 \%$ within the LVR of all samples, G' and G' are presented in Fig. 5c and d, respectively. In emulsions LS 0.75 PC-591 and LS $1.5 \%$ PC-611, G' and G' are comparable; all the other samples have G' > G', indicating a prevalence of the elastic component, with a gel-like behaviour. The FF emulsions present the highest values of G' despite not having the highest viscosity in the flow curves (Fig. 5 a and b), showing the importance of surfactants and microstructure in the rheology of the system, resulting in a more structured network. Indeed this formulation was observed to have the smallest oil droplets, more tightly packed, and very good storage stability. On the other hand, LSF and NSF have a less organized microstructure due to the reduction of surfactants, which is compensated with the continuous phase structure given by the network of solid cellulosic particles. A stronger structuring effect is achieved with BC:CMC, amongst the cellulosic materials. As observed with the rheological profiles, compared to Avicels, BC:CMC has a superior stabilization effect, and PC-591 performs better than PC611. The recorded mechanical spectra (G' and G') show a behaviour nearly independent from the frequency (with almost flat slope), which is typical of gel samples, except for samples with Avicel PC-611. The slight dependence observed can be ascribed to the predominance of noncovalent bonds in the network, independently from the strength of the network (for purely chemically bonded polymers, the slope would be zero) [36].

Similar observations were recorded after 30 days of storage (Appendix A.1), with small deviations in all samples, probably due to stabilization of the formulation components (surfactants) to an equilibrium state. In particular, BC:CMC samples showed a constant behaviour from the beginning and good reproducibility from the followed protocol. Despite the larger oil droplets in the BC:CMC samples, these emulsions were spatially stabilized by the BC fibre 3D network and the effect of a higher viscosity, which effectively prevented the lipid globules from coalescing through all the storage period tested.

\subsubsection{No surfactant formulations}

The NSF produced with BC:CMC exhibited again the same viscosity pattern as the correspondent low surfactant formulations (Fig. 6a and b), also indicating that the continuous phase properties are ruling the overall emulsion rheological behaviour. Similarly, G' and G"' profile showed a solid-like behaviour predominance (Fig. 6c), with a very slight increase in the moduli values over the tested angular frequency range, indicative of the predominance of physical bonds in the entangled network, as in the correspondent LSF. Also in this case there were no changes in the average rheological parameters of the samples after a 30 day storage period (Appendix A.2). Therefore, in terms of rheological properties, there is a good indication towards the feasibility of totally replacing the use of surfactants by BC:CMC particles in this type of cosmetic formulations.

\subsubsection{Three interval thixotropy test}

3ITT methodology was applied to investigate the emulsion's timedependent behaviour. The test begins at a low shear rate, in ideally near-rest conditions; in the second stage, a high shear rate is applied in order to break the internal structure; finally, in the third stage, the material is allowed to recover/rebuild its initial structure under lowshear (near-rest) conditions. In this way, this methodology can show how much of the material's structure can be recovered, and how quickly $[38,39]$. Thixotropic materials undergo structure breakdown under shear and take time to rebuild at rest. This feature allows cosmetics to 
(a)

FS1

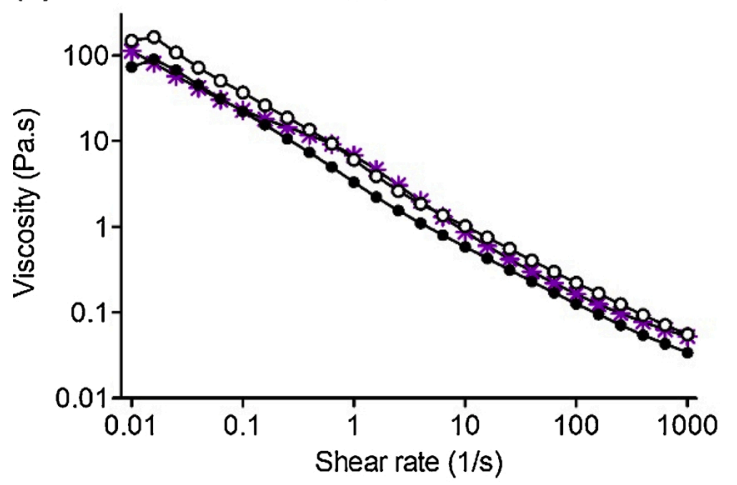

(b) $\quad \mathrm{FS3}$

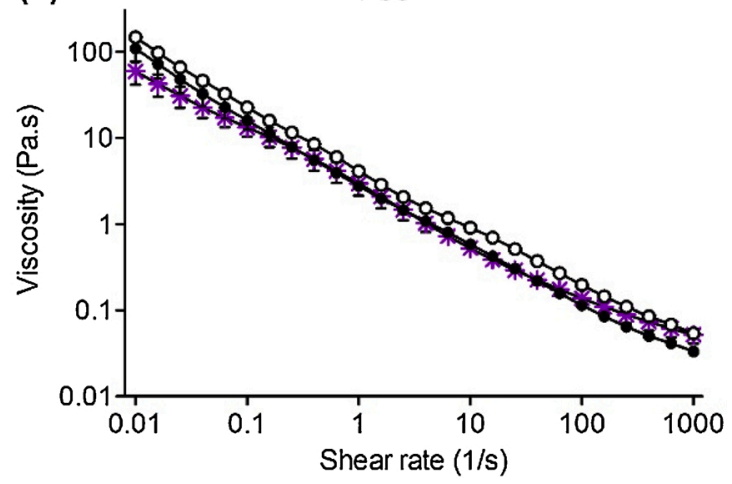

* FF $\rightarrow \mathrm{NS} 0.5 \mathrm{BC}: \mathrm{CMC}$

-o- NS 0.75 BC:CMC

(c)

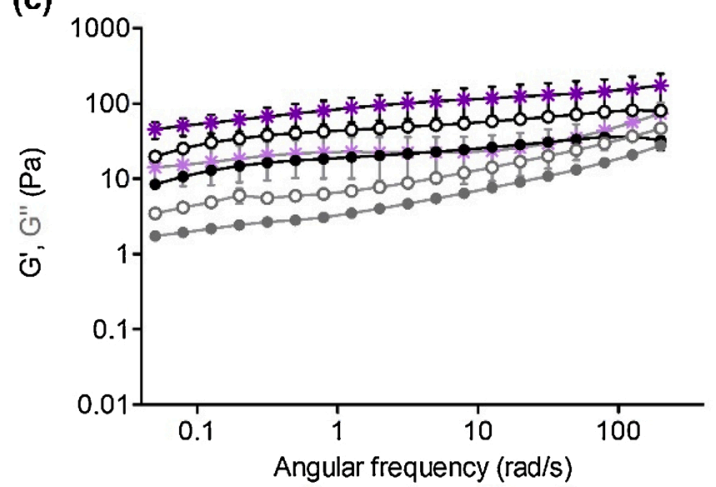

* G'FF

- $\mathrm{G}^{\prime} \mathrm{NS} 0.5 \mathrm{BC}: \mathrm{CMC}$

-o- G' NS 0.75 BC:CMC

* G"FF

$\rightarrow$ G" NS 0.5 BC:CMC

-o- G" NS 0.75 BC:CMC

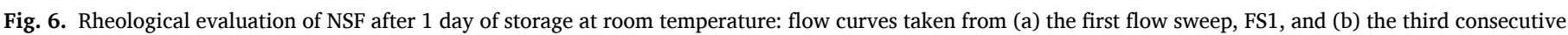

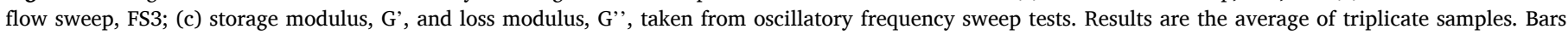
represent standard deviation.

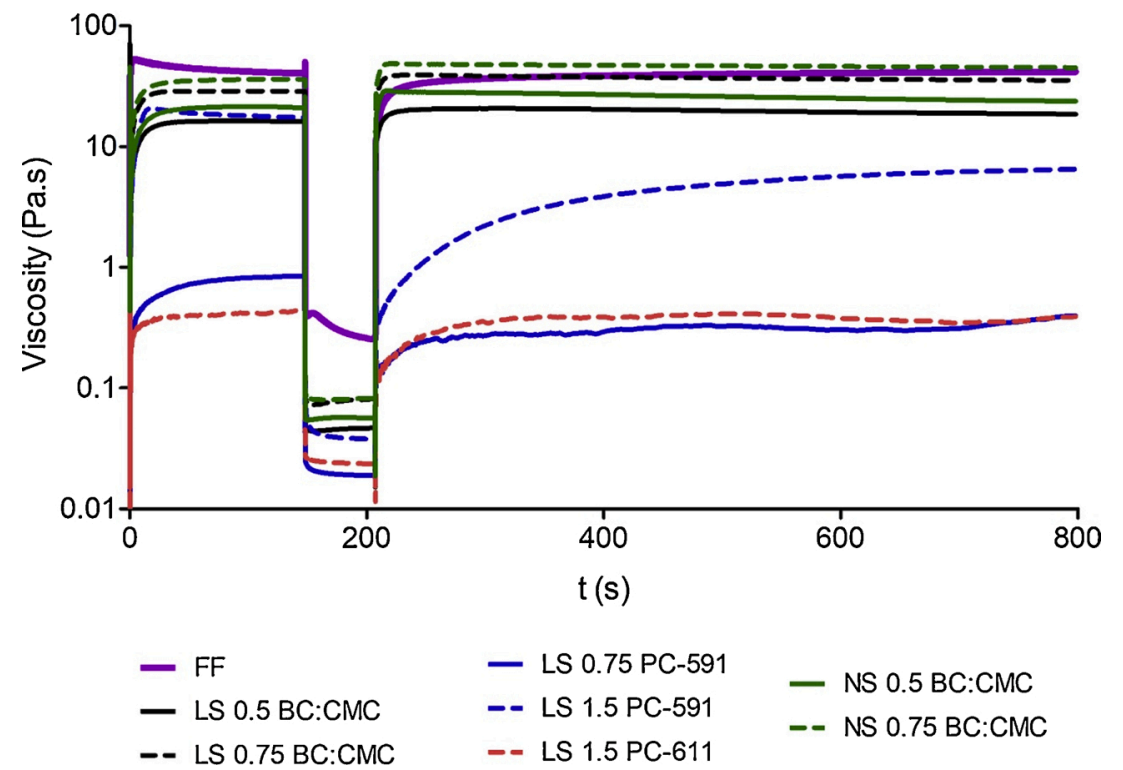

Fig. 7. Three interval thixotropy flow curves of the cosmetic cream analogues after 1 day of storage, at room temperature. 
flow smoothly as they are rubbed in, and recover their structure to stop flowing once they sit on the skin.

Regarding the results from Fig. 7, the FF cream shows some degree of thixotropy as it takes some time but manages to rebuild the structure and regain its initial viscosity. Recovery of the steady-state viscosity achieved at the end of interval 1, approximately 40 Pa.s, is reached back within $255 \mathrm{~s}$ of interval 3 (at $\mathrm{t}=465 \mathrm{~s}$ ).

BC: CMC emulsions (LS and NS) show a lower thixotropy degree than FF. The internal structured network of dispersed BC:CMC quickly reaches the equilibrium state between breakdown and rebuild when the shear conditions are changed. The recovery was almost immediate (within approximately $1 \mathrm{~s}$ ) and resulted in a slightly higher viscosity than that observed in the first stage, possibly due to an improved dispersion of the fibres after the high shear period [38]. Avicel creams, on the other hand, showed greater lag in the structure recovery phase and did not return to the initial viscosity within the analysed time interval.

\subsubsection{Viscoelastic behaviour under oscillatory temperature cycles}

Fig. 8 displays the behaviour of the cosmetic cream analogues when submitted to temperature cycles. The most evident result in this dynamic temperature test concerns the surfactant-stabilized FF cream, which undergoes drastic variation in the elastic behaviour, G', when subjected to temperature changes. The sample shows high G' values at low temperatures and low G' at higher temperatures (from a practical point of view, this facilitates application of the product to the warm skin, since the solid-like behaviour becomes less pronounced). At the final $10{ }^{\circ} \mathrm{C}$ plateau, after 2 complete cycles, G' remained high and close to the initial value. There is a slight rise tendency towards the end of the graphic that is common to all samples, therefore most probably due to some degree of structure rearrangements under oscillation, or to the method itself (for example, a more appropriate solvent trap could eliminate this effect). On the other hand, on the second $50{ }^{\circ} \mathrm{C}$ plateau, the G' dropped relatively to the first cycle, laying below the unit. Lower temperatures do not seem to impact much on the viscoelasticity, but cycles of high temperatures may cause loss of stability in the FF cream. From this result, it is also expected that organoleptic characteristics will vary with temperature, potentially changing the way the product feels on the hand or is applied to the skin. Contrarily to this, the samples that were totally or partially stabilized with cellulose solid particles maintain a much more constant behaviour despite the temperature, indicating a good storage stability even in uncontrolled environments and the maintenance of organoleptic properties regardless of the temperature.

\subsection{Texture assessment}

Texture analysis is a tool based on the conversion of quantitative force measurements into qualitative organoleptic parameters, thus making an approximation to the sensory description of the materials. Two different tests were made: a penetration test, using a cylindrical probe with a small contact area, and a back-extrusion test using a large contact area probe. The penetration test is less sensitive to differences in force; the back-extrusion test, having greater contact area with the sample, besides being more sensitive can also provide information on more textural parameters.

The penetration test would be the equivalent to dipping a finger inside a cream. The main parameters measured in this test are the firmness (maximum force that the sample exerts on the probe during penetration), and consistency (total work performed by the probe to penetrate the sample to a defined depth).

In the back-extrusion test, these same parameters are measured in the downward movement; in the upward movement, the sample's cohesiveness (the maximum absolute force measured during probe withdrawal), and the respective work - the index of viscosity - are determined. This last test is applied to the almost entirety of the sample in the container. With these tests it is possible to replicate different kinds of cosmetic cream handling, more gentle or more intense (corresponding to gentle finger dipping or extrusion of a large amount of sample), and to validate whether the differences between each sample and the control can be felt regardless of the kind or intensity of handling.

Looking at the texture analysis results from Fig. 9 it is noticeable that, overall, the FF cream has higher mean values for all the parameters, which can be attributed to the microstructure. A narrower size distribution, tighter packing of emulsion droplets and the structuring effect of the surfactant in a more consistent network offer a resistance to movement that translates in higher forces necessary for the probes' motion. However, the standard deviations are also much larger. The properties of the surfactant-stabilized FF, besides being dependent on the
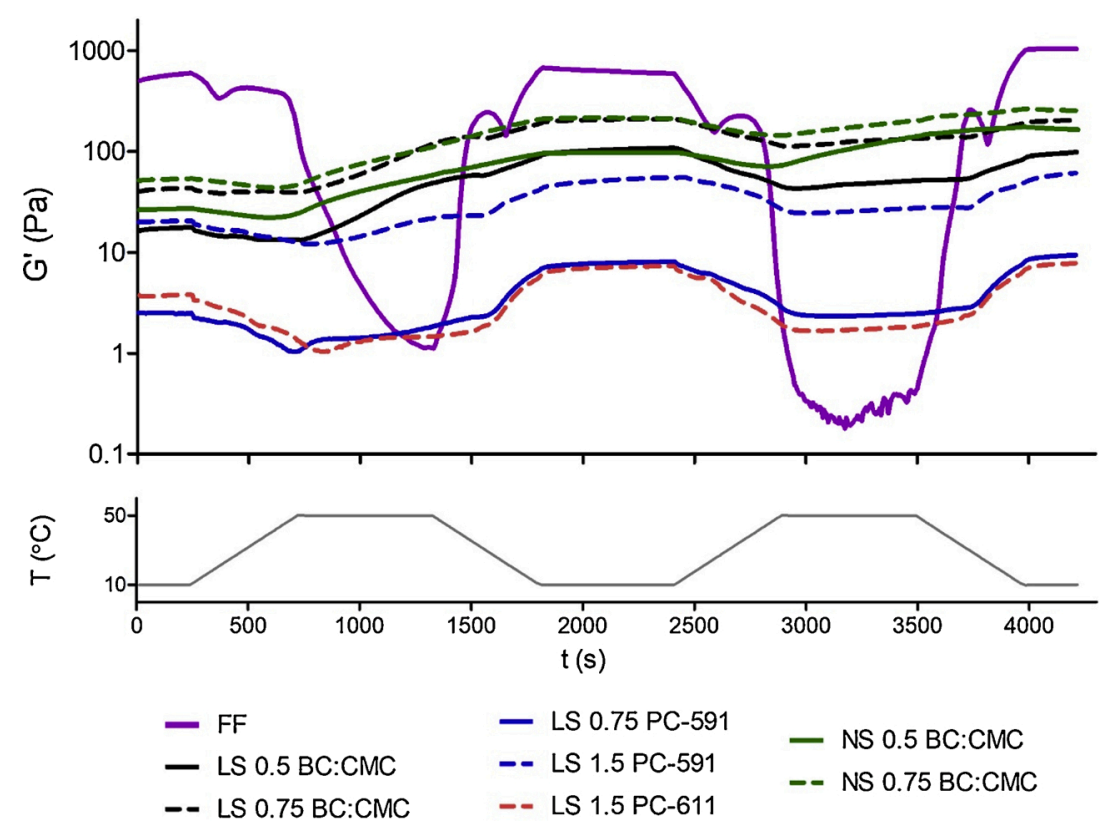

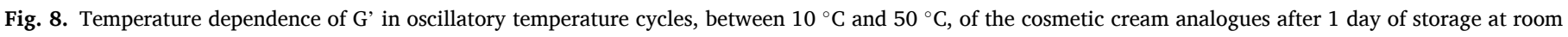
temperature. 
(a)

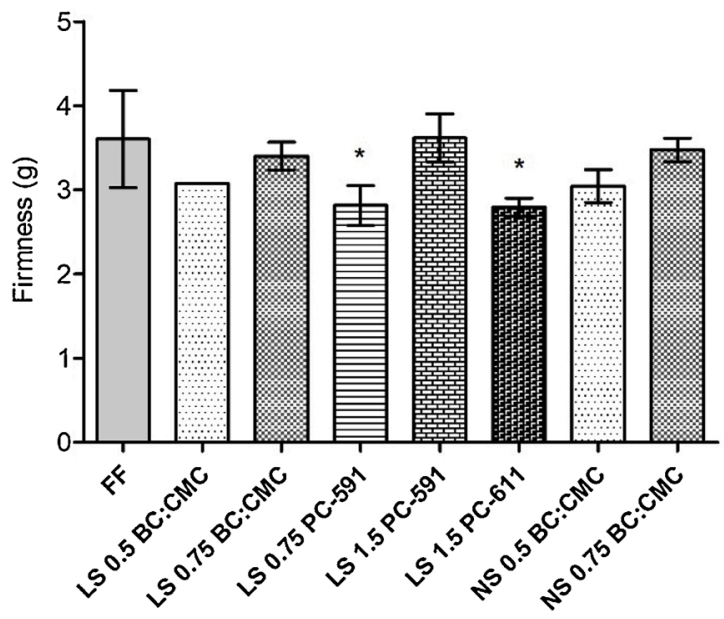

(c)

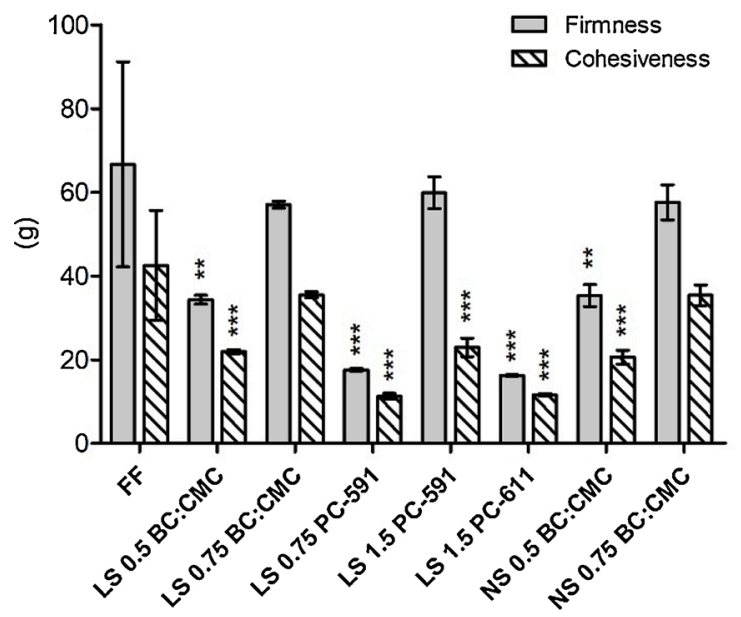

(b)

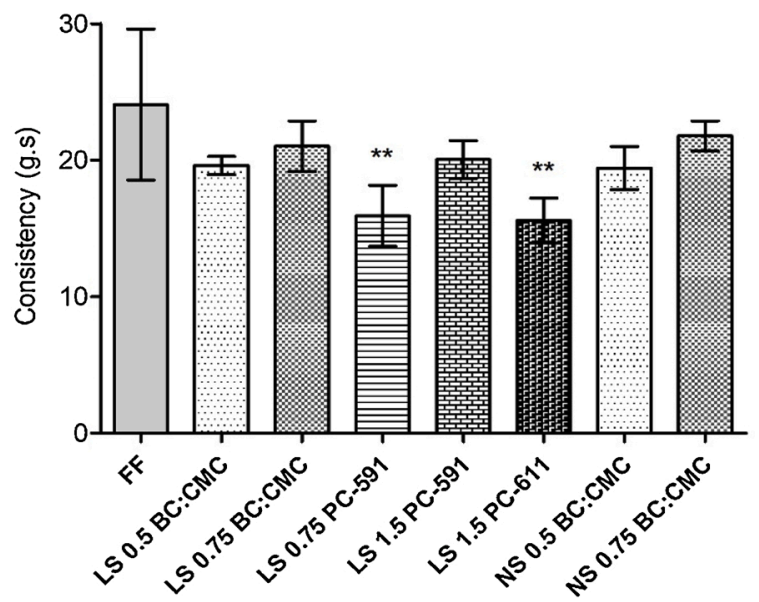

(d)

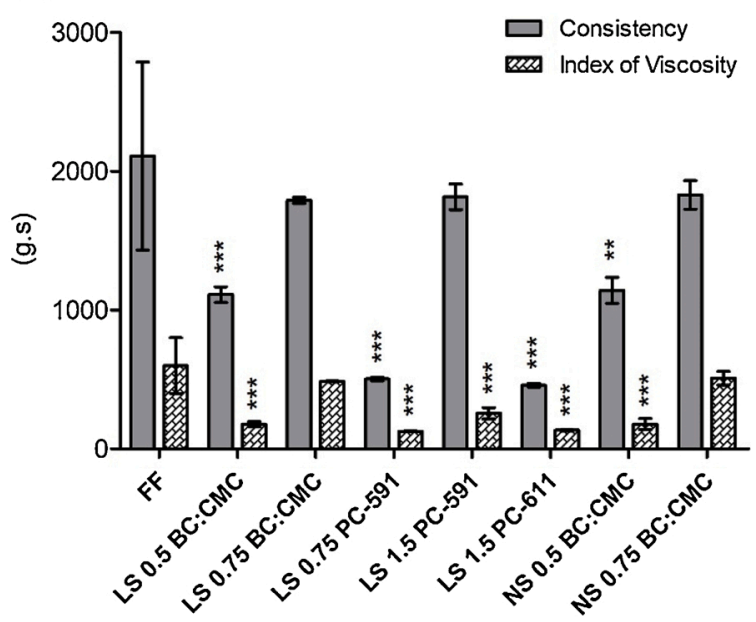

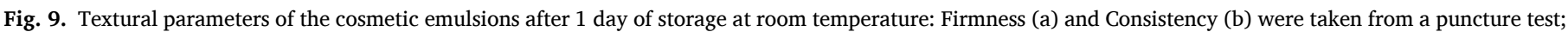

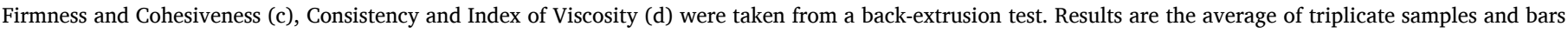

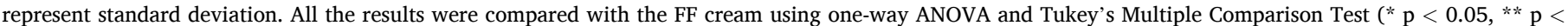
$0.01, * * * \mathrm{p}<0.001)$.

production parameters like temperature and agitation, are also time dependent and could reach an equilibrium stage (in terms of chemical and steric interactions) later in time, meaning the deviations could be smaller on a later analysis. This cannot be shown directly since texture analysis was not performed after the 30-day storage period, but is inferred from the rheology results (Fig. 5 and Appendix A.1), where a drop in the standard deviation values is observed from 1 to 30 days after preparation. On the other hand, the stability, rheological and textural properties of the polymer-stabilized creams (LSF and NSF) were not dependent on the microstructure alone, but more on the cellulose type and concentration, resulting in smaller deviations and more consistent results. This is further demonstrated by comparing the emulsions of LS 0.5 BC:CMC and LS 0.75 BC:CMC with their NS counterparts, where the physical properties between matching concentrations are similar in every test performed, although different production processes were employed (the samples without surfactants were simply homogenized at room temperature). This can actually be a major advantage in terms of manufacture, since it does not require high energy expenses to heat large volumes, and in terms of formulation it allows for the incorporation of heat-sensitive active ingredients.

Regarding the sensory properties in more detail, in terms of firmness in the back-extrusion tests (Fig. 9c), 3 samples showed no significant differences from the control, consistent with the viscosity profiles of these samples, in particular from the first flow rate sweep: the LS and NS 0.75 BC:CMC emulsions, and LS 1.5 PC-591. The same is observed in the consistency results from back-extrusion (Fig. 9d). Although the absolute values are not directly comparable, the penetration test showed similar firmness and consistency tendencies (Fig. 9a and b), leading to the same conclusions. However, relative differences (and thus statistical significance) are much higher for the back extrusion tests, both due to the much higher area of contact between the plunger and the sample and to the much larger sample volume moved during the test. Concerning the cohesiveness and index of viscosity parameters (Fig. 9c and d), the LS and NS 0.75 BC:CMC also show similar values to the control ( $\mathrm{p}>0.05)$; on the contrary, the LS 1.5 PC-591 creams display lower values ( $\mathrm{p}<$ 0.05), deviating from the FF in these parameters. In the dynamic viscosity measurements performed in the rheometer, PC-591 samples' viscosity profile was also below the FF and $\mathrm{BC}$ :CMC ones, particularly for the third flow sweep step. Further, consistency parameters correlate well with the mechanical spectra (G' and G'), presented in Figs. 5 and 6 and with the "solid-like" character and types of bonds involved in the emulsion network. In fact, both bacterial and plant derived celluloses, namely MCC, are known to create three-dimensional networks [8,9,27] which present an obstacle to the introduction of the probe in the sample, thus increasing the necessary force - resulting in measurable firmness and consistency. However, MCC networks might need higher 
concentrations and more time to rebuild after shearing [7], explaining why less force is needed in the retrieval of the probe - lower cohesiveness and index of viscosity. This explanation of lower structural integrity or cohesion can also apply to the lower values of G' and G', and the decrease in the viscosity profile from the first to the third flow-sweep of LS 1.5 PC-591, as was previously explained.

Overall, the LS and NS emulsions prepared with $0.75 \%$ BC:CMC consistently showed similar results to the FF cream. Formulations with the MCC Avicel PC-591 needed a higher concentration to achieve the same firmness and consistency, still showing lower cohesiveness. The low viscosity MCC Avicel PC-611 fell short to the FF in all assessed parameters.

\section{Conclusions}

A BC:CMC formulation was used for the first time to produce a cosmetic cream, allowing a significant or even total elimination of the need for a chemical surfactant. The utilized BC:CMC is a dry powder which can be quickly incorporated in the formulation, not requiring high energy or long mixing periods to achieve good stabilizing and thickening results. From the results of this work, BC:CMC's technological potential seems to be high and might fulfill a market need for more natural cosmetic ingredients, without compromising the performance or sensorial attributes of the formulations.

Samples prepared with $0.75 \%$ BC:CMC consistently showed similar results to the FF control cream, in all the performed tests (visual observation, storage stability, rheology and texture), suggesting that it was possible to mimic relevant rheological and textural properties in formulations with reduced or no surfactants. BC:CMC also performed better than the MCCs used as benchmark, Avicel PC-591 and PC-611, requiring lower concentrations while stabilizing a formulation without surfactants. Although involving a derivatized cellulose, this formulation is clearly advantageous as compared to chemical surfactants, which are claimed to present some skin-irritation issues.

\section{CRediT authorship contribution statement}

D. Martins: Investigation, Methodology, Formal analysis, Writing original draft. C. Rocha: Methodology, Resources, Writing - review \& editing. F. Dourado: Conceptualization, Validation, Writing - review \& editing, Supervision. M. Gama: Conceptualization, Writing - review \& editing, Supervision.

\section{Declaration of Competing Interest}

The authors report no declarations of interest.

\section{Acknowledgements}

This study was supported by the Portuguese Foundation for Science and Technology (FCT) under the scope of the strategic funding of UIDB/ 04469/2020 unit and BioTecNorte operation (NORTE-01-0145-FEDER000004) funded by the European Regional Development Fund under the scope of Norte2020 - Programa Operacional Regional do Norte. Daniela Martins also gratefully acknowledges FCT for the PhD scholarship, reference SFRH/BD/115917/2016.

\section{Appendix A. Supplementary data}

Supplementary material related to this article can be found, in the online version, at doi:https://doi.org/10.1016/j.colsurfa.2021.126380.

\section{References}

[1] S. Bom, J. Jorge, H.M. Ribeiro, J. Marto, A step forward on sustainability in the cosmetics industry: a review, J. Clean. Prod. 225 (2019) 270-290, https://doi.org/ 10.1016/j.jclepro.2019.03.255.

[2] F. Freitas, V.D. Alves, M.A.A. Reis, Bacterial polysaccharides: production and applications in cosmetic industry, Polysaccharides (2014) 1-2241, https://doi.org/ 10.1007/978-3-319-03751-6_63-1.

[3] B.A. Khan, N. Akhtar, H.M.S. Khan, K. Waseem, T. Mahmood, A. Rasul, M. Iqbal, H. Khan, Basics of pharmaceutical emulsions: a review, Afr. J. Pharm. Pharmacol. 5 (2011) 2715-2725, https://doi.org/10.5897/AJPP11.698.

[4] D.K.F. Santos, R.D. Rufino, J.M. Luna, V.A. Santos, L.A. Sarubbo, Biosurfactants: multifunctional biomolecules of the 21st century, Int. J. Mol. Sci. 17 (2016) 1-31, https://doi.org/10.3390/ijms17030401.

[5] R.P. Vianna-Filho, C.L.O. Petkowicz, J.L.M. Silveira, Rheological characterization of $\mathrm{O} / \mathrm{W}$ emulsions incorporated with neutral and charged polysaccharides, Carbohydr. Polym. 93 (2013) 266-272, https://doi.org/10.1016/j. carbpol.2012.05.014.

[6] M.C. Adeyeye, A.C. Jain, M.K.M. Ghorab, W.J. Reilly, Viscoelastic evaluation of topical creams containing microcrystalline cellulose/sodium carboxymethyl cellulose as stabilizer, AAPS PharmSciTech 3 (2002) 1-10, https://doi.org/ 10.1208/pt030208.

[7] V.S. Rudraraju, C.M. Wyandt, Rheological characterization of Microcrystalline Cellulose/ Sodiumcarboxymethyl cellulose hydrogels using a controlled stress rheometer: part I, Int. J. Pharm. 292 (2005) 53-61, https://doi.org/10.1016/j. ijpharm.2004.10.011.

[8] Y. Yaginuma, T. Kijima, Effect of $\mathrm{pH}$ on rheological properties of microcrystalline cellulose dispersions, J. Dispers. Sci. Technol. 27 (2006) 365-370, https://doi.org/ 10.1080/01932690500359483.

[9] G.H. Zhao, N. Kapur, B. Carlin, E. Selinger, J.T. Guthrie, Characterisation of the interactive properties of microcrystalline cellulose-carboxymethyl cellulose hydrogels, Int. J. Pharm. 415 (2011) 95-101, https://doi.org/10.1016/j. ijpharm.2011.05.054.

[10] T. Kondo, P. Rytczak, S. Bielecki, Bacterial nanoCellulose characterization, in: M. Gama, F. Dourado, S. Bielecki (Eds.), Bact. Nanocellulose - From Biotechnol. to Bio- Econ., Elsevier B.V., 2016, pp. 59-71.

[11] R.J. Moon, A. Martini, J. Nairn, J. Simonsen, J. Youngblood, Cellulose Nanomaterials Review: Structure, Properties and Nanocomposites, 2011, https:// doi.org/10.1039/c0cs00108b.

[12] M. Gama, F. Dourado, S. Bielecki, Bacterial Nanocellulose: From Biotechnology to Bio-Economy, Elsevier B.V., 2016, https://doi.org/10.1017/ CBO9781107415324.004.

[13] S.M. Keshk, Bacterial cellulose production and its industrial applications, J. Bioprocess. Biotech. 04 (2014), https://doi.org/10.4172/2155-9821.1000150.

[14] H. Ougiya, K. Watanabe, Y. Morinaga, F. Yoshinaga, Emulsion-stabilizing effect of bacterial cellulose, Biosci. Biotechnol. Biochem. 61 (1997) 1541-1545, https://doi. org/10.1271/bbb.61.1541.

[15] P. Paximada, E. Tsouko, N. Kopsahelis, A.A. Koutinas, I. Mandala, Bacterial cellulose as stabilizer of o/w emulsions, Food Hydrocolloids 53 (2016) 225-232, https://doi.org/10.1016/j.foodhyd.2014.12.003.

[16] X. Zhai, D. Lin, D. Liu, X. Yang, Emulsions stabilized by nanofibers from bacterial cellulose: new potential food-grade Pickering emulsions, Food Res. Int. 103 (2018) 12-20, https://doi.org/10.1016/j.foodres.2017.10.030.

[17] I.F. Almeida, T. Pereira, N.H.C.S. Silva, F.P. Gomes, A.J.D. Silvestre, C.S.R. Freire, J.M. Sousa Lobo, P.C. Costa, Bacterial cellulose membranes as drug delivery systems: an in vivo skin compatibility study, Eur. J. Pharm. Biopharm. 86 (2014) 332-336, https://doi.org/10.1016/j.ejpb.2013.08.008.

[18] T. Amnuaikit, T. Chusuit, P. Raknam, P. Boonme, Effects of a cellulose mask synthesized by a bacterium on facial skin characteristics and user satisfaction, Med. Devices Evid. Res. 4 (2011) 77-81, https://doi.org/10.2147/MDER.S20935.

[19] N. Chunshom, P. Chuysinuan, S. Techasakul, S. Ummartyotin, Dried-state bacterial cellulose (Acetobacter xylinum) and polyvinyl-alcohol-based hydrogel: an approach to a personal care material, J. Sci. Adv. Mater. Devices. 3 (2018) 296-302, https://doi.org/10.1016/j.jsamd.2018.06.004.

[20] K. Ludwicka, M. Jedrzejczak-Krzepkowska, K. Kubiak, M. Kolodziejczyk, T. Pankiewicz, S. Bielecki, Medical and cosmetic applications of bacterial nanocellulose, in: M. Gama, F. Dourado, S. Bielecki (Eds.), Bact. Nanocellulose From Biotechnol. to Bio-Economy, Elsevier B.V., 2016, pp. 145-165.

[21] Y. Numata, L. Mazzarino, R. Borsali, A slow-release system of bacterial cellulose gel and nanoparticles for hydrophobic active ingredients, Int. J. Pharm. 486 (2015) 217-225, https://doi.org/10.1016/j.ijpharm.2015.03.068.

[22] Z.-F. Yang, N.A. Morrison, T.A. Talashek, D.F. Brinkmann, D. DiMasi, Y.L. Chen, US 2007/019777 A1 - Bacterial Cellulose-containing formulations, 2007.

[23] B.P. Heath, T.W. Coffindaffer, K.E. Kyte, E.D. Smith, S.D. McConaughy, Wo 2011/ 019876 A2 - Personal Cleansing Compositions Comprising a Bacterial Cellulose Network and Cationic Polymer, 2011.

[24] F. Tournilhac, R. Lorant, US 6534071 B1 - Composition in the Form of an Oil inWater Emulsion Containing Cellulose FIBRILS, and Its Uses, Especially Cosmetic Uses, 2003.

[25] R. D'Ambrogio, D.A. Peru, J.E. Gambogi, K.M. Kinscherf, D. Patel, R. Tavares, WO 2011/056951 A1 - Microfibrous Cellulose Having a Particle Size Distribution for Structured Surfactant Compositions, 2011.

[26] M. Caggioni, R. Ortiz, F.A. Barnabas, R.V. Nunes, J.A. Flood, F. Corominas, US 8716213 B2 - Liquid Detergent Composition Comprising an External Structuring System Comprising a Bacterial Cellulose Network, 2014. 
[27] D. Martins, B. Estevinho, F. Rocha, F. Dourado, M. Gama, A dry and fully dispersible bacterial cellulose formulation as a stabilizer for oil-in-water emulsions, Carbohydr. Polym. 230 (2019) 115657, https://doi.org/10.1016/j. carbpol.2019.115657.

[28] D. Martins, D. de Carvalho Ferreira, M. Gama, F. Dourado, Dry Bacterial Cellulose and Carboxymethyl Cellulose formulations with interfacial-active performance: processing conditions and redispersion, Cellulose 9 (2020), https://doi.org/ 10.1007/s10570-020-03211-9.

[29] L. Gilbert, G. Savary, M. Grisel, C. Picard, Predicting sensory texture properties of cosmetic emulsions by physical measurements, Chemometr. Intell. Lab. Syst. 124 (2013) 21-31, https://doi.org/10.1016/j.chemolab.2013.03.002.

[30] L. Gilbert, C. Picard, G. Savary, M. Grisel, Rheological and textural characterization of cosmetic emulsions containing natural and synthetic polymers: relationships between both data, Colloids Surf. A Physicochem. Eng. Asp. 421 (2013) 150-163.

[31] L. Gilbert, V. Loisel, G. Savary, M. Grisel, C. Picard, Stretching properties of xanthan, carob, modified guar and celluloses in cosmetic emulsions, Carbohydr. Polym. 93 (2013) 644-650, https://doi.org/10.1016/j.carbpol.2012.12.028.

[32] E. Dickinson, Hydrocolloids and emulsion stability, in: G.O. Philips, P.A. Williams (Eds.), Handb. Hydrocoll., 2 ed, Woodhead Publishing, 2009, pp. 23-49, https:// doi.org/10.1533/9781845695873.23.
[33] Y. Chevalier, M.A. Bolzinger, Emulsions stabilized with solid nanoparticles: pickering emulsions, Colloids Surf. A Physicochem. Eng. Asp. 439 (2013) 23-34 https://doi.org/10.1016/j.colsurfa.2013.02.054

[34] B.P. Binks, Particles as surfactants - similarities and differences, Curr. Opin. Colloid Interface Sci. 7 (2002) 21-41.

[35] T. Winuprasith, M. Suphantharika, Properties and stability of oil-in-water emulsions stabilized by microfibrillated cellulose from mangosteen rind, Food Hydrocolloids 43 (2015) 690-699, https://doi.org/10.1016/j. foodhyd.2014.07.027.

[36] J.W. Goodwin, R.W. Hughes, Rheology for Chemists: An Introduction, Royal Society of Chemistry, 2000, https://doi.org/10.5860/choice.38-3918.

[37] J.F. Steffe, Rheological Methods in Food Process Engineering, second edition, Freeman Press, 1996.

[38] B. Basnet, W.Y. Jang, J.G. Park, I.S. Han, T.Y. Lim, H.M. Lim, I.J. Kim, Impact of $\mathrm{SiC}$ colloidal suspension properties for the fabrication of highly porous ceramics, J. Ceram. Process. Res. 18 (2017) 634-639, https://doi.org/10.36410/ jcpr.2017.18.9.634.

[39] O.S. Toker, S. Karasu, M.T. Yilmaz, S. Karaman, Three interval thixotropy test (3ITT) in food applications: a novel technique to determine structural regeneration of mayonnaise under different shear conditions, Food Res. Int. 70 (2015) 125-133, https://doi.org/10.1016/j.foodres.2015.02.002. 